

\title{
Optimized Protein-Protein Interaction Network Usage with Context Filtering
}

Natalia Pietrosemoli, Maria Pamela Dobay

\section{To cite this version:}

Natalia Pietrosemoli, Maria Pamela Dobay. Optimized Protein-Protein Interaction Network Usage with Context Filtering. Computational Cell Biology. Methods in Molecular Biology, pp.33-50, 2018, 10.1007/978-1-4939-8618-7_2 . hal-02861812

\section{HAL Id: hal-02861812 \\ https://hal.science/hal-02861812}

Submitted on 19 Jun 2020

HAL is a multi-disciplinary open access archive for the deposit and dissemination of scientific research documents, whether they are published or not. The documents may come from teaching and research institutions in France or abroad, or from public or private research centers.
L'archive ouverte pluridisciplinaire HAL, est destinée au dépôt et à la diffusion de documents scientifiques de niveau recherche, publiés ou non, émanant des établissements d'enseignement et de recherche français ou étrangers, des laboratoires publics ou privés.

$$
\text { Copyright }
$$




\title{
Chapter 2
}

\section{Optimized Protein-Protein Interaction Network Usage with Context Filtering}

\author{
Natalia Pietrosemoli and Maria Pamela Dobay
}

\begin{abstract}
Protein-protein interaction networks (PPIs) collect information on physical—and in some cases-functional interactions between proteins. Most PPIs are annotated with confidence scores, which reflect the probability that a reported interaction is a true interaction. These scores, however, do not allow users to isolate interactions relevant in a particular biological context. Here, we describe solutions for performing context filtering on PPIs to allow biological data interpretation and functional inference in two publicly available PPIs resources (HIPPIE and STRING) and in the proprietary pathway analysis tool and knowledge base Ingenuity Pathway Analysis.
\end{abstract}

Key words Protein-protein interaction networks, Context filtering, Orthogonal text mining resources

\section{Introduction}

Protein-protein interaction networks (PPIs) are typically used in biological interpretation to explain differences among the observed phenotypes, particularly in enrichment analyses and in functional inference via guilt-by-association methods [1]. PPIs can be grouped into physical interaction networks and functional PPIs. Tools which provide physical interaction networks include HIPPIE, which integrates various direct interactions from public and curated databases [2] and STRING, which provides both physical interactions between proteins and functional interactions, such as in signaling cascades [3, 4]. While PPIs built using HIPPIE are derived from experimental results, STRING also incorporates other interactions sources such as text mining, coexpression evidence, co-occurrence evidence, genomic neighborhood (i.e., genes that colocalize in the genome), gene fusion, as well as information from other PPIs databases to infer interactions (Table 1) [3]. Other PPIs that focus on experimentally validated physical interaction information include the Human Protein Reference 
Database (HPRD, [5]), Molecular INTeraction database (MINT, [6]), IntAct [7], and the Biological General Repository for Interaction Datasets (BioGRID, [8]). Both STRING and HIPPIE consolidate information from all these other PPIs and can thus be considered "meta"-PPI resources [9].

With the exception of HIPPIE, which provides options for filtering PPIs by biological context [2, 10], most interaction network tools filter PPIs using confidence scores, which measure the reliability of an interaction as a function of the amount and kind of experimental evidence that supports it.

Other proprietary resources such as Ingenuity Pathway Analysis (IPA, http://www.ingenuity.com) permit creating networks linking query genes. IPA does not present confidence scores, but instead allows users to choose among three different confidence levels: experimentally observed interactions, predicted high and predicted moderate interactions. To start building networks, IPA queries the Ingenuity Knowledge Base $(\mathrm{KB})$ for both interquery interactions and then for interactions with all other objects stored in the KB. Similar to STRING, the KB of IPA is a repository of curated biological interactions and functional annotations created from individually modeled relationships between proteins, genes, complexes, cells, tissues, drugs, and diseases gathered from both public and private biomedical databases. A main extension provided in IPA is the integration of small molecule data and links with databases on diseases and disease biomarkers. A main issue regarding PPI or $\mathrm{KB}$ use in biological interpretation is degeneracy or the lack of concordance among the first-degree neighboring protein vertices (i.e., proteins with a direct connection to the queries used to build the network) retrieved from using different PPI tools, and even different versions of the same PPI tool [9] (see Note 1).

As an example, we show the differences in the network neighborhoods of 22 influenza A virus (IAV) entry factors (Table 1) in different PPI tools (Fig. la). We see that not only are the derived network topologies obtained from using the default settings quite diverse among the different tools, but the sizes of their network neighborhoods and, thus, their extent of overlap, calculated by dividing the total number of overlapping components (edges of vertices) by the number of components of the smaller of two graphs $G_{1}$ and $G_{2}$ being compared, are highly different too (Fig. lb, c). Finally, if we extract the abstracts that are used as evidence for an interaction from PubMed and check the frequency of biological keywords associated with these (see Notes 2 and 3), we see that majority of the interactions are linked to cancer (STRING, Fig. 2a) or signaling in general (STRING and IPA, Fig. 2a, b), and only a small proportion of supporting evidence from literature have been specifically reported in virus infection-related processes. 


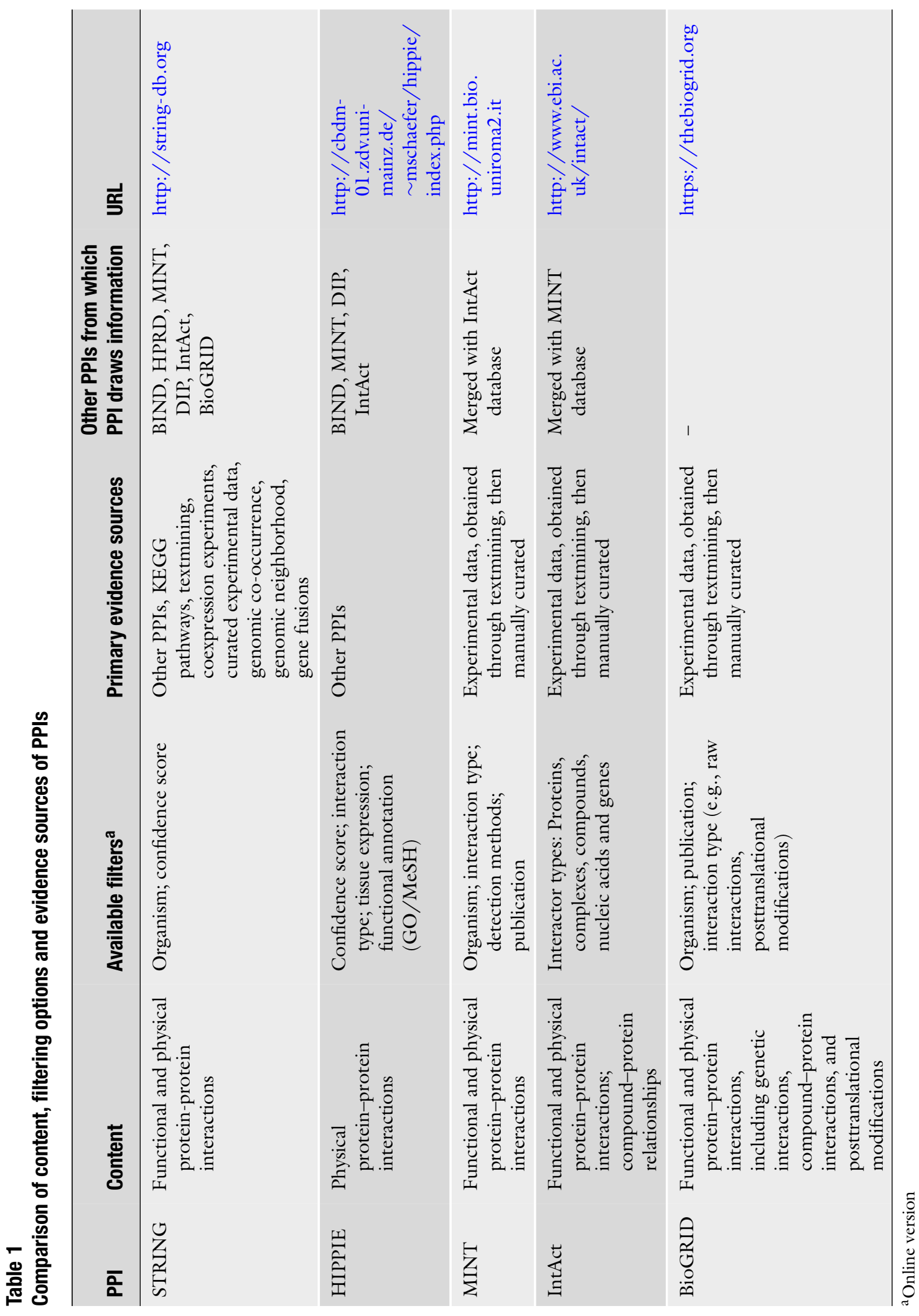


A

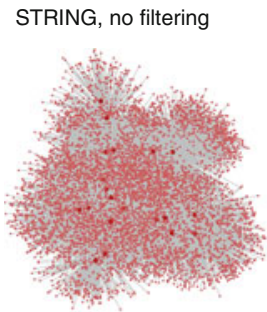

MINT

B Edge overlap

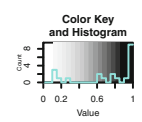

STRING, high confidence

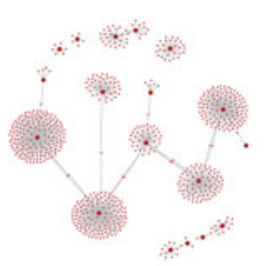

IntAct

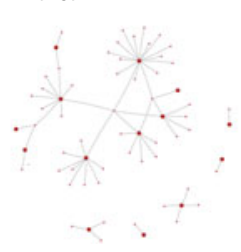

HIPPIE

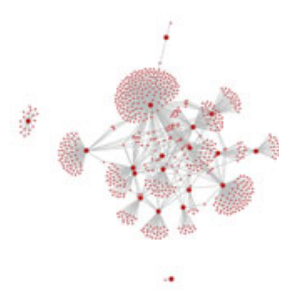

BioGRID

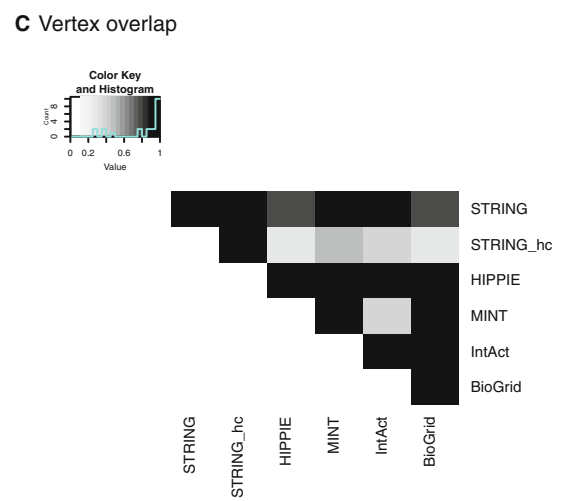

Fig. 1 Network neighborhoods of influenza A virus (IAV) entry factors in different protein-protein interaction networks and IPA (a). Query vertices from the entry screen are shown in dark red. Pairwise network neighborhood overlaps for edges (b) and vertices (c) of all tools except IPA. Overlaps are calculated as the number of vertices or edges in common between the graphs divided by the number of vertices or edges of the smaller graph. A filtered version of STRING restricted to high-confidence edges (STRING_hc, confidence score $>800, A)$-calculated as the fraction of overlapping edges or vertices with respect to the smaller graph-indicates that smaller network neighborhoods are not always completely included in the larger ones

This observation evidences an important bias in the supporting literature [9].

Thus, to maximize the utility of PPI tools, it is necessary to isolate interactions, which are relevant in specific biological contexts. Here we describe how to use context filters in two meta-PPI resources, STRING and HIPPIE, and the proprietary IPA tool. Additionally, we show how to use the R package rentrez (https:// cran.r-project.org/web/packages/rentrez/index.html) to check if the retrieved edges are indeed implicated in the process of interest (i.e., molecules involved in the interactions-incident vertices- 

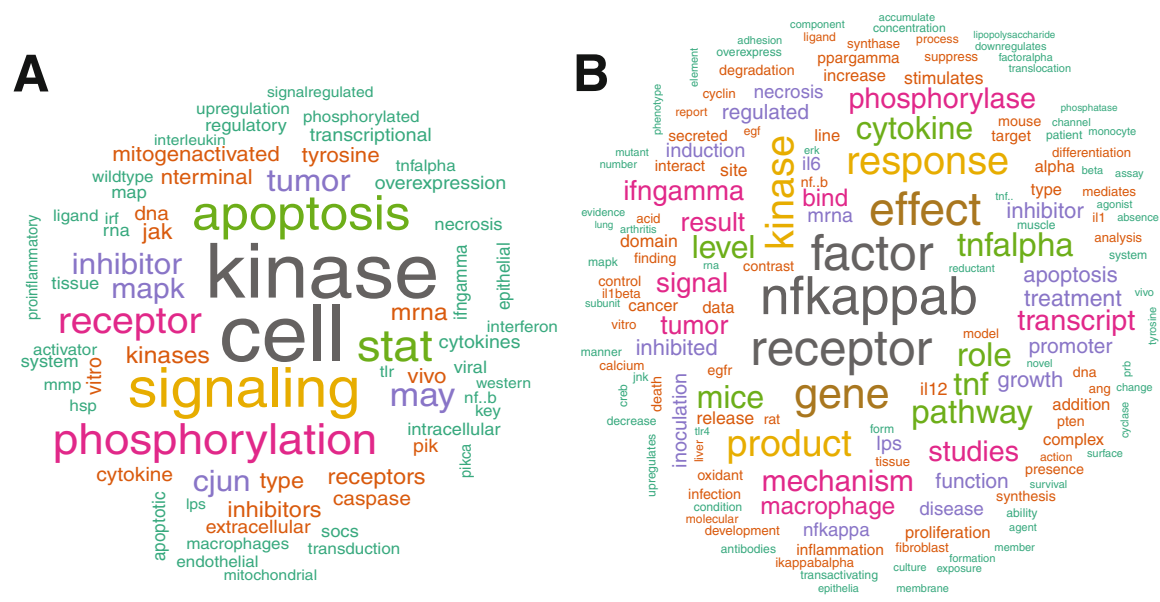

Fig. 2 Most frequently used words from textmining evidence of the entry network neighborhood of the unfiltered STRING network (a) and IPA network (b). Evidence for the STRING network neighborhood is characterized by the predominance of signaling-related terms, and mainly implicates STAT, MAPK, and caspase signaling. For IPA, the implicated signaling pathways terms include the NF-kappa $B$, TNF, and interferon signaling pathways. Note that influenza A hijacks various pathways, including NF-kappa B, PI3K/Akt, MAPK, PKC/PKR, TLR/RIG-I, mTOR, EGFR, and ERK signaling [20], with early signaling being predominantly linked to PKC/PKR [21] and EGFR [22]; nonetheless, information directly linking interactants to mechanical effectors of entry, such as clathrin or caveolin, are not immediately evident from the network neighborhood. Details on how the wordclouds are generated are described in [9]

are comentioned together with the process of interest in a record abstract, see also Subheading 5, Note 2).

\section{Materials}

\subsection{Data}

The following versions of PPIs were used in this analysis:

STRING: v.10, online version and STRINGdb R package (v. 1.14.0).

HIPPIE: online version; for R-based manipulations, we used HIPPIE v.1.8.

IntAct: (ftp://ftp.ebi.ac.uk/pub/databases/intact/current/ all.zip, downloaded in December 2015).

HPRD: Release9_062910.

MINT: 2012-10-29 (last release).

IAV entry factors can be found under the following link: https://github.com/pampernickel/flu_ppi/blob/master/data/ annotations/hgnc.csv

Mappings between HUGO gene nomenclature committe (HGNC) symbols [11] and entrez IDs can be found in this file:

https://raw.githubusercontent.com/pampernickel/flu_ppi/ master/data/annotations/hgnc.csv 


\subsection{Software Packages}

\subsection{Code Repositories}

All analyses were performed using the $\mathrm{R}$ language, version 3.2.3. Analyses were also tested and confirmed to run on $\mathrm{R} 3.2 .2$ and 3.3.3.

Critical code and functions required to reproduce results shown in this book chapter are deposited in: https://github.com/ pampernickel/flu_ppi/blob/master/sample_codes/sampleScripts.r. sampleScripts.r is provided to illustrate vertex- and edge-based filtering. All custom function dependencies are likewise provided in the same repository: https://github.com/pampernickel/flu_ ppi/tree/master/sample_codes/functions

\section{Methods}

\subsection{First-Degree Network Neighborhood Construction and Context Filtering in HIPPIE}

Context filtering has been introduced in HIPPIE in the form of tissue expression, cellular compartment (cc) and biological process (bp) annotations. The following steps describe how a context-filtered first-degree network neighborhood for your query protein(s) in HIPPIE is created:

1. For a single query, input the query protein name on the default HIPPIE tab ("protein query"); for multiple queries, select the network tab and input a list of query proteins (Fig. 3a). Note that in HIPPIE, both the single query and multiple query modes yield the first-degree neighborhoods of the query proteins.

2. Select the output type; the default output is a visualization of the network on the web browser ("show in browservisualization"). If you wish to import the results into an analysis platform (e.g., R software environment), a tab-delimited format (HIPPIE TAB or PSI-MI TAB formats) is recommended.

3. Check all relevant filters (Fig. 3b), which include the interaction type restrictions (interaction type filter), tissue expression localization (tissue filter); and Gene ontology (GO) or Medical Subject Heading ( $\mathrm{MeSH}$ ) annotations (functional filter). GO terms, which are standardized terms ("ontologies") that describe gene function and their relationships [12], are further categorized into biological processes or cellular compartments in HIPPIE. MeSH headings [13] are likewise standardized terms that facilitate indexing of journal articles in the life sciences - and by extension, their content-according to its subject. HIPPIE MeSH terms are restricted to diseaseassociated terms. Note that all functional filters are menus (delineated by a "+" symbol), and clicking on the "+" sign expands the choices to more specific GO or MeSH terms.

4. After selecting filters and output options, click on the "SEARCH" button, located just below the query box. 
A

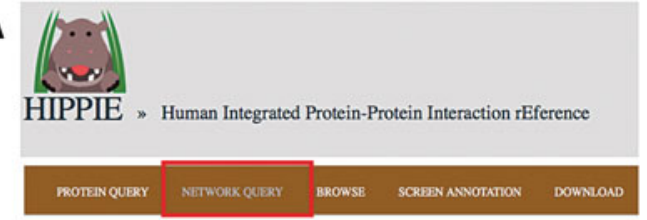

C



B


E
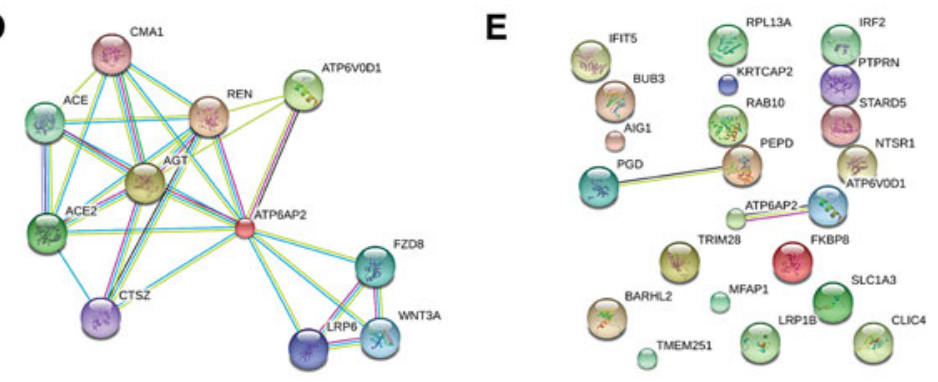

Fig. 3 Context filters in HIPPIE and STRING. Context filtering options in HIPPIE can be accessed in the network query mode (red box, a). The menu of filtering options (b) allows the user to impose various restrictions on the retrieved network neighborhood based on the interaction type, the tissue(s), cellular context ("biological_process") or location ("cellular_component") in which the interaction has been reported, or the diseases in which the interaction has been implicated ("MeSH," not shown). STRING allows searching with default parameters using a single protein name or multiple protein names as input parameters (red boxes, c). Note that querying with a single name retrieves the first-level interactors of the query protein (d), while querying with multiple names retrieves the interaction network between the query proteins, without the inclusion of first-level interactors (e)

5. If you selected a tab-delimited format as an output, results will be automatically downloaded; if you selected the default option, the interaction network is shown on the same window.

(a) In the case of more than ten queries, or queries involving signaling pathway molecules (e.g., MAPK or EGFR), where the number of neighboring vertices is expected to exceed more than 40, the HIPPIE visualization result is not informative. An option to improve the visualization is to download the network neighborhood as a JSON object or tab-delimited text, which can be imported into Cytoscape (see also Note 4), a platform for network visualization and analysis [14]. 


\subsection{First-Degree Network Neighborhood Construction and Context Checking in STRING (Web Interface)}

(b) Options for enrichment analysis on the network neighborhood are offered at this point to check for overrepresented genes linked to diseases, GO biological process, GO molecular functions or to GO cellular compartment. GO term enrichment calculations are done with the PANTHER overrepresentation test, which is based on the binomial distribution, rather than the hypergeometric distribution [15].

STRING allows the submission of single or multiple protein names as queries, and with a medium confidence filter (Fig. 3c). When the query is comprised of a single protein, it returns the first-degree network (Fig. 3d), whereas a query with multiple proteins yields the interactions between these proteins (Fig. 3e). This is different from how single and multiple queries are processed in HIPPIE.

The following steps describe how to check the interactions for a single protein in STRING (for users of the web interface, the procedure is very limited in terms of performance)

1. In the main STRING page, select "Proteins by name" and enter the name of your query. Indicate the species for which you want to retrieve the interactions; alternately, leave the auto-detect option on. Click "search."

2. If the species was specified, the network neighborhood for the query protein is displayed as an image, together with options for data exploration and download; if the species was not specified, you will be first redirected to a page where you can select the [auto-detected] species.

3 . To access the different types of evidence linked to the network neighborhood, click on the "evidence" button below the image. This reveals a list of evidence sources used to build the neighborhood. To specifically check the context of the text mining evidence associated with the network, click on the "textmining" button, which shows excerpts from the abstract or full text where the interacting proteins are comentioned.

4. Alternately, if you want to check the text mining evidence specifically linked to an edge, click on the green lines linking two protein vertices. This opens a popup window that shows the evidence types available for that specific interaction. Click "show" on the text mining evidence box. This redirects you to a page listing the text mining reference(s) specifically associated with that edge.

5. As in HIPPIE, it is possible to run enrichment analyses on the network neighborhood of your query. Click on the "analysis" button below the graphical network to reveal enriched GO terms (biological processes, molecular functions and cellular components), functional pathways (KEGG) protein domains (PFAM and INTERPRO) and enriched interactions. By click- 


\subsection{First-Degree Network Neighborhood Construction and Context Filtering in STRING (STRINGdb R Package)}

3.3.1 Network
Neighborhood
Extraction in STRINGdb
for $R$

ing on a selected enriched term, it is possible to visualize all proteins in the network annotated with this term. You may change the background of the enrichment analysis from the whole genome to just the druggable genome or the kinome.

Context filtering is currently not available in the web versions of STRING, but with the release of STRING for R (STRINGdb, [4]), it is possible to implement script-based workarounds, which are described in detail below. While the methods are recommended for users with basic knowledge of the $\mathrm{R}$ language [16], we provide several running examples that walk the user through the minimum steps in R (and the STRINGdb and igraph R packages) required to extract a context-filtered network (see Note 3, https:// github.com/pampernickel/flu_ppi/blob/master/sample_codes / sampleScripts.r). Note that all the STRING tables and STRING output in $\mathrm{R}$ are converted, where possible, to igraph objects, which allow the direct application of graph algorithms on STRING content.

1. Create a vector of gene names for which you want to get the network neighborhood; to ensure higher chances of identification, use HGNC symbols.

2. Load STRINGdb to a variable (string.db) using the STRINGdb\$new command. Specify the version, organism, and confidence score threshold that you wish to work with. In our example, we use version 10 for Homo sapiens (Taxonomy identifier: 9606), with a score threshold of 0 to include the full network.

3. Load the full STRING graph to your workspace as an igraph object with the getGraph command (string.db\$getGraph).

4. Map your vector of gene names to STRING identifiers using the map command (string.db\$map). Note that the vector of gene names is passed to a custom function, prepareMap to process it in the format that STRING requires. This step returns a data frame (vertexMap in our example) consisting of your original vector of gene names and the corresponding STRING ids.

5. Extract the first-degree neighborhood using the getNeighbors function applied to the STRING identifiers in vertexMap (from Subheading 3.3.1, step 4). Use the STRING graph from Subheading 3.3.1, step $\mathbf{3}$ as the query graph. Convert the final object (a list of all neighbors per query), using the constructGraph function to an igraph object.

6. Get a reverse mapping of the vertex names (currently in the form of STRING identifiers) to HGNC symbols using the string.db\$get_aliases function; select the subset of aliases that are HGNC symbols. 
3.3.2 Vertex-Based Filtering (GO Biological Process)
3.3.3 Edge-Based Filtering (Keyword Filtering of Textmining Evidence)
7. The resulting form of the graph is now ready for GO biological process annotation.

A first step to use a similar vertex filter as in HIPPIE entails the annotation of network components with GO terms in R. In our example, we use the getgos and the nodeToGO functions, which are dependent on the org.Hs.eg.db package (v.3.1.2). For usage, see sampleScripts.r. Note that this method is applicable to any network without GO annotations.

1. Create a vector of GO terms of interest. Given that GO terms can vary widely in specificity, choose a GO term at a level that is specific enough to be informative. In sampleScripts.r, we use the GO bp terms (vesicle-mediated transport) and GO:0060627 (regulation of vesicle-mediated transport) and selected descendants of this GO term (i.e., excluding terms linked to synaptic vesicle transport) for filtering (Fig. 4a).

2. Use the GOBPCHILDREN mapping from the GO.db package to include the descendants of the GO terms of interest; check the names of the descendant terms using the getGOnames function to remove GO daughter terms that fall under the main GO term query, but are not of interest (e.g., GO:1903421, regulation of synaptic vesicle recycling).

3. Using the getNodeAttribute function and a combination of $\mathrm{R}$ base functions, you can check which vertices linked to a query vertex are annotated with a GO term of interest. Only edges connecting query vertices and vertices annotated with a GO term of interest are retained (Fig. 4b).

Most edges in STRING are supported by textmining evidence, which give some contextual information regarding the interactions. The textmining evidence was obtained from the application of a text retrieval and analysis algorithm of STRING run on PubMed abstracts and when available, on full article texts.

1. Create the entry network neighborhood following steps $\mathbf{1 - 5}$ of Subheading 3.3.1 (i.e., up to step where an igraph object is created with STRING identifiers, and not HGNC identifiers).

2. Convert the igraph object in Subheading 3.3.3, step 1. to a data frame, then extract all references linked to each edge using the string.db\$get_pubmed_interaction function. This returns a vector of PubMed and Online Mendelian Inheritance in Man (OMIM) identifiers, when applicable; OMIM identifiers indicate if a protein has been implicated in a genetic phenotype [17].

3. Extract the abstracts linked to these PubMed IDs using entrez_fetch; use XML as the return type; use functions 
A



C
B

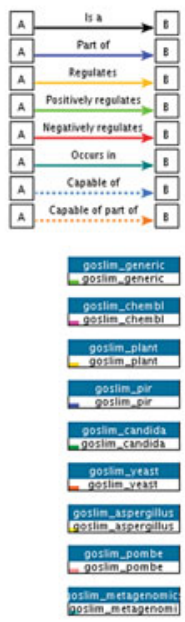

$$
\text { (1) }
$$

.

Go term annotations
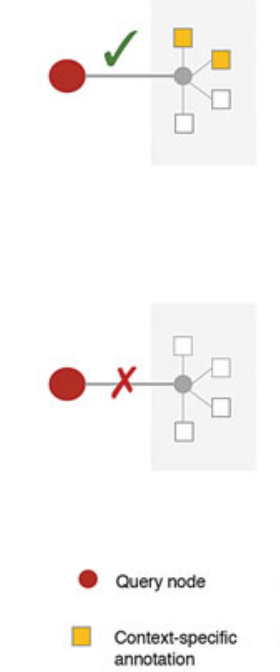

Vertex filtering
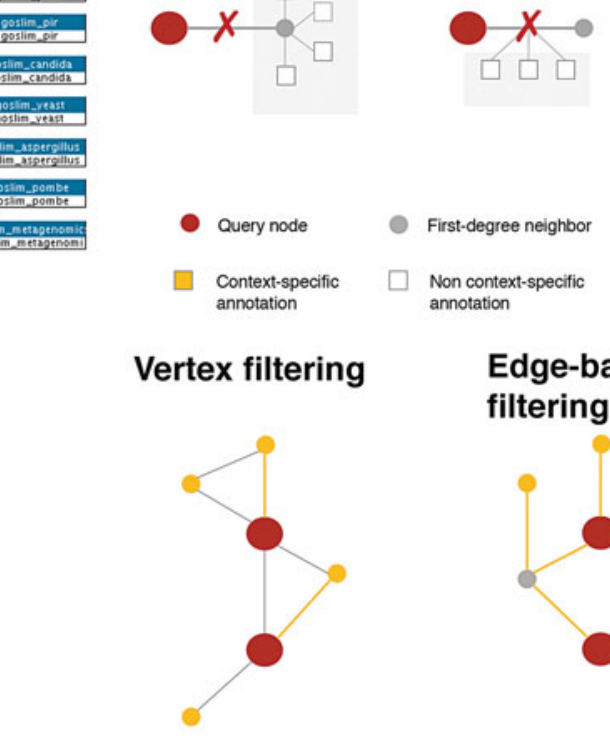

First-degree neighbor

Non context-specific annotation

\section{Edge-associated textmining evidence}



Echovirus 7 entry into polarized intestinal We previlus ncistrint 0 ex

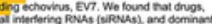


cogative

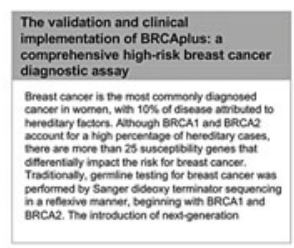

Edge-based filtering
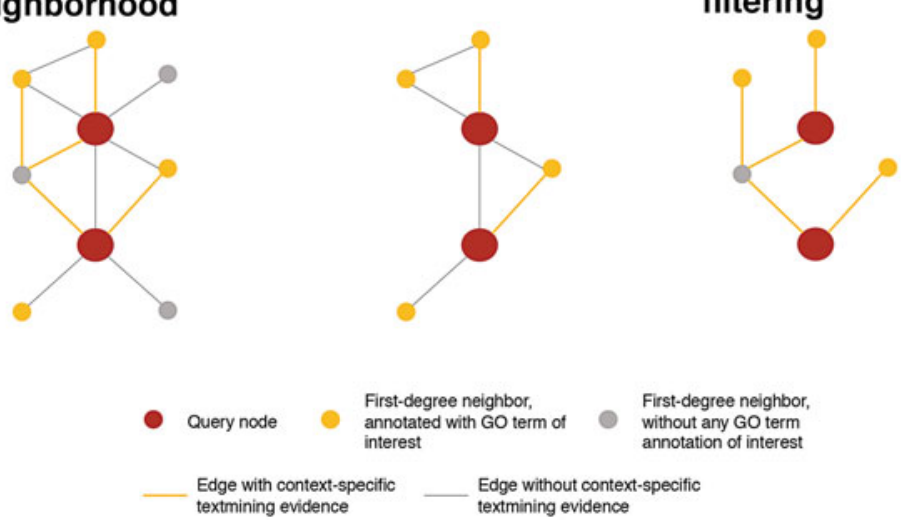

Fig. 4 Vertex- and edge-based context filtering. Examples of entry-related terms (in yellow) in the G0 biological process (bp) hierarchy for the G0 term, regulation of vesicle-mediated transport (G0:0060627, http://www.ebi.ac.uk/QuickGO) (a). Note that term associations are nested, with some terms being less specific than others (e.g., "localization" encompasses more genes than "vesicle-mediated transport"). Context filtering using G0 annotations (b) results in the retention of edges with relevant G0 term annotations (b, top left) connected to a query vertex; analogously, edges supported by textmining evidence mentioning keywords of interest are retained (b, top right). Note that context filtering can still result in degenerate solutions (c), but all retained vertices and edges are at least implicated in the process of interest

$\mathbf{x m l T r e e P a r s e}$ and the getAbstract to retrieve the abstracts as a character vector.

4. Create a vector of keywords to search for in the retrieved abstracts, then check the frequency of occurrence of each of the keywords in the abstracts. Note that the keywords can be patterns rather than full keywords (e.g., "endosom" 


\subsection{First-Degree \\ Network \\ Neighborhood \\ Construction \\ and Context \\ Filtering in IPA}

instead of "endosome," which matches both "endosome" and "endosomal"). Retain edges that are supported by abstract(s) with a minimum number of keywords (Fig. 4b).

Note that vertex- and edge-based filtering may still result in degenerate solutions (Fig. 4c). Nonetheless, unlike in the case of confidence score filtering, it is at least certain that retained elements would be restricted to those linked to the process of interest.

Alternatively, a recently released Cytoscape application, stringApp (http://apps.cytoscape.org/apps/stringapp, compatible with Cytoscape 3.3) also allows the direct import of STRING data into Cytoscape [18]. The application allows three main query modes, namely protein names (as described in Subheading 3.2), disease, and PubMed query. The PubMed query option mirrors results that can be obtained in Subheading 3.3.3. The disease mode yields the top proteins linked to a disease based on information from the DISEASE database [19]. Finally, if the information is available for the organism from which a query protein is from, information regarding the subcellular localization and tissue expression are also indicated and can be used to contextualize the network.

Ingenuity Pathway Analysis is a proprietary software that allows modeling and analysis of biological systems. There are several ways of building a network in IPA (Stand alone version: Version 28,820,210; Building krikkit Date 2016-09-24), but the general workflow consists of the following steps:

1. Format your input list of molecules according to the IPA standards. All formatting is done outside IPA in a spreadsheet program (e.g., Excel) to produce the input file.

2 . The header must consist of only one row, and there should be no empty cells. Unlike in HIPPIE or STRING where the only data required are protein names, IPA allows also associating measurement values (up to three) for each molecule (fold changes and $p$-values for expression data, variant loss/gain values, phosphorylation ratio, differential metabolomics data, etc.).

3. Mixed identifier types (Gene Symbol HGNC, Ensembl, Entrez, Uniprot, Unigene, etc.) are allowed and mapped to a common identifier

4. Upload your molecule list file as a flat file (.cvs, .txt, .tsv) or an excel file (.xls) using the file menu: "File"; "Upload dataset"; "Formatted data file". Define the upload settings, including the "file format" (Flexible Format), the "column header" (Yes) and the "identifier type," which is usually automatically detected (e.g., HGNC gene symbol). If using data from microarrays, the platform details need to be specified 
for identifier mapping, otherwise the platform is set to "Not specified/applicable."

5. Select columns of the input file to use in the analysis: "ID column," and optionally, the observation column(s) (including potential threshold columns such as $p$-value and expression value) and their corresponding measurement type.

6. Choose "Save the dataset," as all datasets are automatically annotated when they are uploaded into IPA. It is important to verify the mapping of the dataset in order to identify mapped and unmapped identifiers (IDs). The annotated dataset comprises several columns describing the uploaded molecules that provide specific details such as gene ID, Symbol, Entrez Gene Name, a unique subcellular location (column Location; which can be: cytoplasm, nucleus, plasma membrane or other), one functional gene family (column Type(s); which can be: enzyme, G-protein coupled receptor, ion channel, kinase, ligand-dependent nuclear receptor, peptidase, transcription regulator, transporter or other) and association with drugs (column Drug(s)).

7. Run the core analysis on the annotated dataset by choosing "Analyze/Filter Dataset"; "Core Analysis."

8. Define all the filter settings (most of them have default values which may be kept):

9. "General settings Reference set": Population of genes to consider as a reference or background set to be used for ranking the statistical significance (i.e., $p$-value calculation) in the enrichment analyses. Options from the IPA KB include the genes or proteins only (default), metabolites only, or a combination of genes and metabolites. The user can also specify the background based on the microarray platforms or a user-uploaded dataset.

10. Type of relationships to consider (that affect networks and upstream regulator analysis), which could be direct (default) and indirect. Direct relationships imply a physical interaction between two molecules (e.g., a kinase and its known substrate, or a drug and its protein target). Indirect relationships may occur through intermediates (e.g., the relationship between a chemokine receptor and the gene whose expression it induces via downstream signaling).

11. Parameters for network construction: option to include (default) or exclude endogenous chemicals, number of molecules per network (35 by default), and number of networks per analysis ( 25 by default).

12. Specify the vertex types to be included: this parameter allows to build or filter the network based on the specific types of molecules selected (e.g., biological drug, chemical 
(kinase inhibitor, chemical drug, protease inhibitor), cytokines, enzymes, growth factors, microRNAs, transcription regulators, transmemebrane receptors).

13. Specify the data sources to be used: allows contextual data analysis filtering. The list includes primary PPI databases (e.g., BioGRID, IntAct, see Table 1), ontological annotations (e.g., GO, OMIM), and other sources (COSMIC database for mutations, DrugBank) from which data in network reconstruction should be derived.

Other contextual filtering parameters include:

(a) "Confidence": this parameter determines the confidence level on the reported interactions. It allows three different types: experimentally observed (default); high or moderate confidence.

(b) "Species": by selecting items in this filter, you are specifying to consider only ortholog genes of the selected species. Broad categories are mammal (human, mouse, rat) and uncategorized. This parameter includes an additional filter: "stringent" or "relaxed." The first option will return only those molecules and relationships relevant to the selected species. The relaxed filter will match molecules that contain an ortholog that includes the selected species. This option filters on the orthologs, but not the relationships between the orthologs.

(c) "Tissues and Cell Lines": filter for genes expressed in a particular tissue or cell line. Currently, IPA includes data from 21 different cell lines, mainly cancer cell lines. Again, there are two filtering choices: "Stringent" and "Relaxed" which are analogous to those for the "Species" filter.

(d) "Mutations" findings from the Ingenuity Knowledge Base are now available for inclusion in the generated networks. Such findings involve a mutant form of at least one of the genes. You can choose to exclude mutant findings using this filter-the default is to include relationships that involve wild type and mutant forms of genes.

The results of the analysis consist in all the molecules that interact with other molecules in the Ingenuity Knowledge Base, which are identified as "Network Eligible" molecules and serve as "seeds" for generating networks. Network Eligible molecules are combined into networks that maximize their specific connectivity (i.e, their interconnectedness with each other relative to all molecules they are connected to in the Ingenuity $\mathrm{KB}$ ). Additional molecules from the Ingenuity $\mathrm{KB}$ are used to connect two or more smaller networks by merging them into a larger one. 
Results can be exported in three tab-delimited files (references, molecules, relationships). The relationships file details all interactions (experimental or a combination of experimental and predicted), as well as the nature of interactions (activation, inhibition, etc.). The references file contains IDs linked to various data sources (selected in step 6e) from which relationships were derived. Unlike in STRING, references cannot be associated oneto-one with the edges.

\section{Summary and Outlook}

PPIs contain a wealth of information that may be used in functional inference. Given the varied contexts from which the information is derived, however, it is useful to be able to filter PPIs according to the context in which each interaction is reported. The context can be defined in terms of common ontological annotations of the vertices, including cellular localization and biological function. Here, we show how to take advantage of information - in particular, the supporting literature associated with each edgeto isolate subnetworks whose elements have been reported in a set of biological contexts of interest.

\section{Notes}

1. We have noted that disparities in network-neighborhoods can occur between different versions of the same PPI [9]. It is clearly good practice to check the consistency of your results whenever a new version of a PPI is released by checking, for instance, if the change occurred at the level of one of the primary databases, or (in the case of STRING) if there are differences in the textmining sources used as edge evidence.

2. One way of evaluating the relevance of retrieved network neighborhoods without performing preliminary experiments is to check for articles that either mention the vertices of a network neighborhood together with keywords of interest (i.e., the vertices have been implicated in the biological context of interest, but the interaction in the PPI might be novel, as could be the case in vertex-based filters), or check for articles that comention the incident vertices together with keywords specific to a biological process of interest (i.e., the interaction has been documented in the biological context of interest). In the GO-filtered STRING network for virus entry, which is comprised only of vertices and edges that have been previously annotated with a GO term specific for entry (Fig. 4a) we 
have checked the frequency at which each retained vertex is comentioned with one of eight keywords of interest that were not used in filtering. To efficiently perform such checks, one can use PubMed e-utilities (https://www.ncbi.nlm.nih.gov/ books/NBK25501/) or wrappers, which are a set of functions that simplify exploration and access of NCBI databases, such as rentrez. A typical search in e-utilities is defined by the database and the query term; it is also convenient to set the maximum number of retrieved terms. This is done by specifying the following fields in your query on https://eutils.ncbi.nlm.nih. gov/entrez/eutils/esearch.fcgi?:

(a) db: NCBI database on which to perform the search; to search for abstracts, pubmed should be specified.

(b) term: search term

(c) retmax: number of retrieved matches that should be returned; note that in the case of PubMed abstracts, hits are ordered both by time (i.e., more recent articles first) and relevance.

Typing the following on your web browser, for instance, yields the PubMed IDs of the 1000 most recent articles on influenza from PubMed in an XML file: https://eutils.ncbi.nlm.nih. gov/entrez/eutils/esearch.fcgi? db=pubmed\&term=influenza\& retmax $=1000$.

3. For users with experience in $\mathrm{R}$ programming, using rentrez is a more flexible option. To retrieve the same results as above, the following command yields an entrez list object:

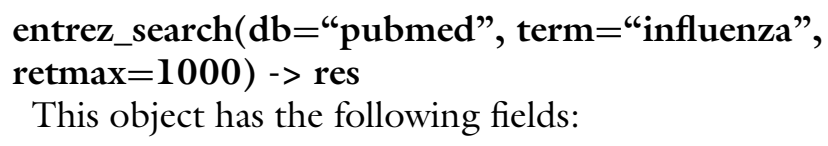

(a) ids: PubMed IDs of the relevant results, limited to the number specified in retmax; can be obtained by typing res\$ids

(b) count: total number of records in PubMed that match the query term

(c) retmax: number of retrieved matches that should be returned.

(d) Query translation: in case the search term matches any ontological term in PubMed, the search is expanded to include all these terms. In this example, the query "influenza" is translated into "influenza, human" $[\mathrm{MeSH}$ Terms] OR ("influenza"[All Fields] and "human"[All Fields]) OR "human influenza["All Fields] OR "influenza” [All Fields] 
Additional examples of the use of rentrez to check the availability of information regarding pairs of proteins reported in an interaction network are shown in Example 3 of sampleCodes.r.

4. There are various open-source tools that can be used for network visualization and analyses, including Cytoscape (http:// www.cytoscape.org), Graphviz (http://www.graphviz.org), and Gephi (https://gephi.org), which have graphical user interfaces. For users with more experience in programming, igraph (http://igraph.org) is an efficient analysis tool that is available in $\mathrm{R}$, Python, and $\mathrm{C} / \mathrm{C}++$.

\section{Acknowledgments}

This work was supported by the Swiss Initiative in Systems Biology, SystemsX, through a fellowship $(2013 / 137)$ provided to M.P.D.

\section{References}

1. Rual JF, Venkatesan K, Hao T, HirozaneKishikawa T, Dricot A, Li N et al (2005) Towards a proteome-scale map of the human protein-protein interaction network. Nature 437:1173-1178

2. Schaefer MH, Fontaine JF, Vinayagam A, Porras P, Wanker EE, Andrade-Navarro MA (2012) HIPPIE: integrating protein interaction networks with experiment based quality scores. PLoS One 7:e31826

3. von Mering C, Jensen LJ, Snel B, Hooper SD, Krupp M, Foglierini M et al (2005) STRING: known and predicted protein-protein associations, integrated and transferred across organisms. Nucleic Acids Res 33:D433-D437

4. Franceschini A, Szklarczyk D, Frankild S, Kuhn M, Simonovic M, Roth A et al (2013) STRING v9.1: protein-protein interaction networks, with increased coverage and integration. Nucleic Acids Res 41:D808-D815

5. Peri S, Navarro JD, Amanchy R, Kristiansen TZ, Jonnalagadda CK, Surendranath $\mathrm{V}$ et al (2003) Development of human protein reference database as an initial platform for approaching systems biology in humans. Genome Res 13:2363-2371

6. Zanzoni A, Montecchi-Palazzi L, Quondam M, Ausiello G, Helmer-Citterich M, Cesareni G (2002) MINT: a molecular INTeraction database. FEBS Lett 513:135-140

7. Hermjakob H, Montecchi-Palazzi L, Lewington C, Mudali S, Kerrien S, Orchard S et al
(2004) IntAct: an open source molecular interaction database. Nucleic Acids Res 32:D452D455

8. Stark C, Breitkreutz BJ, Reguly T, Boucher L, Breitkreutz A, Tyers M (2006) BioGRID: a general repository for interaction datasets. Nucleic Acids Res 34:D535-D539

9. Dobay MP, Stertz S, Delorenzi M (2017) Context-based retrieval of functional modules in protein-protein interaction networks. Brief Bioinform

10. Schaefer MH, Lopes TJ, Mah N, Shoemaker JE, Matsuoka Y, Fontaine JF et al (2013) Adding protein context to the human protein-protein interaction network to reveal meaningful interactions. PLoS Comput Biol 9:e1002860

11. Gray KA, Yates B, Seal RL, Wright MW, Bruford EA (2015) Genenames.org: the HGNC resources in 2015. Nucleic Acids Res 43:D1079-D1085

12. Ashburner M, Ball CA, Blake JA, Botstein D, Butler H, Cherry JM et al (2000) Gene ontology: tool for the unification of biology. The gene ontology consortium. Nat Genet 25:2529

13. Beyerly E (1962) New medical subject heading lists: a comparative review of American and soviet works. Bull Med Libr Assoc 50: 196-202

14. Shannon P, Markiel A, Ozier O, Baliga NS, Wang JT, Ramage D et al (2003) Cytoscape: 
a software environment for integrated models of biomolecular interaction networks. Genome Res 13:2498-2504

15. Mi H, Muruganujan A, Casagrande JT, Thomas PD (2013) Large-scale gene function analysis with the PANTHER classification system. Nat Protoc 8:1551-1566

16. R Development Core Team (2014) R: a language and environment for statistical computing. the R Foundation for Statistical Computing, Vienna

17. McKusick VA (2007) Mendelian inheritance in man and its online version, OMIM. Am J Hum Genet 80:588-604

18. Szklarczyk D, Morris JH, Cook H, Kuhn M, Wyder S, Simonovic M et al (2017) The STRING database in 2017: quality-controlled protein-protein association networks, made broadly accessible. Nucleic Acids Res 45:D362D368

19. Pletscher-Frankild S, Palleja A, Tsafou K, Binder JX, Jensen LJ (2015) DISEASES: text mining and data integration of disease-gene associations. Methods 74:83-89

20. Gaur P, Munjhal A, Lal SK (2011) Influenza virus and cell signaling pathways. Med Sci Monit 17:RAl48-RAl54

21. Ludwig S, Planz O, Pleschka S, Wolff T (2003) Influenza-virus-induced signaling cascades: targets for antiviral therapy? Trends Mol Med 9:46-52

22. Eierhoff T, Hrincius ER, Rescher U, Ludwig S, Ehrhardt C (2010) The epidermal growth factor receptor (EGFR) promotes uptake of influenza a viruses (IAV) into host cells. PLoS Pathog 6:e1001099 